\title{
Modified method of conductometric data analysis to calculate the degree of ionization and conductivity of micelles
}

\author{
O. S. Zueva ${ }^{1, *}$ \\ ${ }^{1}$ Kazan State Power Engineering University, Kazan, Russia
}

\begin{abstract}
Methods for calculation of specific conductance of ions and micelles and the degree of micelle ionization using conductometric data in various approximations of the Debye - Hückel - Onsager theory were considered. The analysis of the existing calculation methods was carried out to identify their drawbacks and to suggest ways of their elimination. The calculation method of the micellar parameters on the basis of conductometric data using micellar size was modified, and a new formula for determining the degree of micelle ionization was obtained. All calculations using the modified method were performed in the first and the second approximations, and the newly obtained values of the micellar parameters are in greater agreement with the results of other studies. Based on the calculations performed, it was shown that the contribution of micelles to the total conductivity of micellar solution cannot be neglected, since at high concentrations the contribution of micelles exceeds the contribution of counterions and can exceed $50 \%$.
\end{abstract}

\section{Introduction}

The variety of practical use of aqueous surfactant solutions in power engineering, electronics and other technical applications [1-3], provides the basis for a comprehensive study of these materials by various methods. Surfactant solutions belong to organized solutions due to the special properties of surfactant molecules that tend to self-association and formation of ordered structures (for example, micelles). The ability to control and manage nanostructural changes in the ways of organizing surfactant molecules both inside the solution and on the interface surfaces can give a new impulse to the use of such media in power engineering and electronics.

The control of the occurring changes can be carried out by various methods including conductometry methods. However, for accuracy of the conclusions the correct interpretation of the data is required. Conductometric data is particularly important in the case of surfactants applying in the electrolyte composition of energy-saving devices, since the directed selection of an appropriate electrolyte requires data on the electrical conductivity (mobility) of ions and micelles as well as on the charge and ionization degree of the formed micelles and their contribution to the total conductivity of solution.

The purposes of this work were the following: analyzing existing computational methods; modifying a calculation method for specific conductance of ions and micelles; determining the degree of micelle ionization using conductometric data in the first and the second approximations of the Debye - Hückel - Onsager theory and estimating the contribution of micelles to the total conductivity of micellar solution.

\section{Basic relations}

At low concentrations (in premicellar region) the number of charged particles in ionic surfactant solution is composed of the number of surfactant ions and counterions. When a critical micelle concentration $\left(\mathrm{CMC}, C_{\mathrm{CMC}}\right)$ is reached, surfactant ions begin to form associates called micelles. In the region close to the $\mathrm{CMC}$ associates have a spherical shape and contain the certain number $N$ (the aggregation number) of hydrocarbon chains with polar parts oriented from the core. The outer shell of micellar core consists of potential-determining ions and carries an electric charge, partially compensated by appearance of a double ion layer. The number of counterions in the dense part of the double layer (the Stern layer) is determined by the degree of counterion binding $(\beta)$ to micelles. According to various estimates this number ranges from $60 \%$ to $90 \%$ of the surfactant ions number in associate $(\beta=0.6 \div$ $0.9)$ [4]. Other counterions are bonded weaker with the micellar associate and are distributed in the diffuse part of the double layer. The degree of micelle ionization $\alpha=$ $1-\beta$ determines the charge $q_{\mathrm{M}}$ of micelle (of micellar associate with the dense part of the double layer):

$$
q_{\mathrm{M}}=\alpha Z \text { e } N=Z e(1-\beta) N,
$$

here $\mathrm{Z}$ is charge number of the surface ionic groups, $e$ is the elementary electric charge. A significant difference should be noted in determining the coefficients $\alpha$ and $\beta$

\footnotetext{
* Corresponding author: ostefzueva@mail.ru
} 
for the same surfactant both by different methods and by different ways of calculation.

In premicellar region the specific conductance of electrolyte solution $\kappa(C)$ is determined by value of ion charge and the number of ions carrying electricity, and migration rates or electrical mobility, sometimes also called the conventional mobility of both positive $u_{+}$and negative ions $u$ - in the medium under the action of the electric field gradient. For a binary solution of 1-1 electrolyte $\left(Z=Z_{+}=Z_{-}=1\right)$ at the concentration $C$ and the dissociation degree $\alpha_{0}$, the dependence of the electrical conductivity on concentration is:

$$
\kappa(C)=\alpha_{0} e N_{\mathrm{A}}\left(u_{+}+u_{-}\right) C=\alpha_{0} F\left(u_{+}+u_{-}\right) C=a C .
$$

Here $F=e N_{\mathrm{A}}$ is the Faraday constant related to the Avogadro number $N_{\mathrm{A}}$. The numerical values of counterion and surfactant ion mobilities vary due to difference in their sizes. In the framework of the assumption that ionic mobilities are independent on concentration, the $\kappa(C)$ plot corresponds to a straight line passing through the $C=0$ point, $a$ is the tangent of the angle of inclination of a line.

At concentrations exceeding $\mathrm{CMC}$, the specific conductance of surfactant dispersions is determined by the presence of another type of mobile charged particles - micelles. It is believed that the contribution to conductivity is provided by free surfactant ions and by counterions that are not associated with micelle or distributed in a diffuse part of the double ionic layer, as well as micelles themselves. Redistribution of carriers and their partial aggregation lead to a change in the course of dependence $\kappa(C)$ :

$$
\begin{gathered}
\kappa(C)=\alpha_{\mathrm{o}}\left\{e N_{\mathrm{A}}\left(u_{-}+u_{+}\right) C_{\mathrm{CMC}}+\right. \\
\left.\left.q_{\mathrm{M}} N_{\mathrm{A}} U_{\mathrm{M}}\left(C-C_{\mathrm{CMC}}\right) / N+e N_{\mathrm{A}} \alpha u_{+}\left(C-C_{\mathrm{CMC}}\right)\right]\right\},
\end{gathered}
$$

where $U_{\mathrm{M}}$ is the mobility of micelles (here anionic surfactants are considered for definiteness, in the case of cationic surfactants the signs "+" and "-" in the indices should be swapped). Given that the effective charge of micelle is determined by relation (1), we have

$$
\begin{gathered}
\kappa(C)=\alpha_{\mathrm{o}} F\left[\left(u_{-}+u_{+}\right) C_{\mathrm{CMC}}+\right. \\
\left.\alpha\left(U_{\mathrm{M}}+u_{+}\right)\left(C-C_{\mathrm{CMC}}\right)\right]=a C_{\mathrm{CMC}}+b\left(C-C_{\mathrm{CMC}}\right) .
\end{gathered}
$$

It should be noted that in the framework of the assumption that the mobility of ions and micelles is independent on concentration (which is considered valid in the initial region of micelles existence), the specific conductance $\kappa(C)$ is also described by a straight line, but its inclination with respect to the $C$ axis changes ( $b$ is the tangent of the angle of inclination of line $\kappa(C)$ in the micellar region). It no longer passes through the point $C$ $=0$.

In order to use the available reference data, the specific conductance is usually expressed not through ion mobilities, as in relation (2), but through the values of equivalent electrical conductivities of ions and counterions $\lambda_{ \pm}=F u_{ \pm}$, which identical their molar conductivities in binary 1-1 electrolyte solutions [5]. For considered electrolytes with the dissociation degree $\alpha_{0}=$ 1 , the relations (2) and (4) can be written as

$$
\begin{gathered}
\kappa(C)=\left(\lambda_{+}+\lambda_{-}\right) C=a \cdot C . \\
\kappa(C)=\left[\left(\lambda_{+}+\lambda_{-}\right) C_{\mathrm{CMC}}+\right. \\
\left.\alpha\left(\lambda_{\mathrm{M}}+\lambda_{+}\right)\left(C-C_{\mathrm{CMC}}\right)\right]=a \cdot C_{\mathrm{CMC}}+b\left(C-C_{\mathrm{CMC}}\right),
\end{gathered}
$$

For a micellar concentration range the equivalent conductivity of micelles $\lambda_{\mathrm{M}}=F U_{\mathrm{M}}$ was also used.

Ion molar (equivalent) conductivities are well studied in the theory of solutions. It is known that at the infinite dilution they increase to their limiting values $\lambda_{0+}$ and $\lambda_{0-}$, which are called the limiting molar (equivalent) conductivities of ions. Their values for many types of ions at certain temperatures can be found in reference books [6].

To establish the relationship between conductivity, structural, and diffusion parameters of ions, ions are considered as solid spheres of radius $r$ with charge $q$ moving in a solvent with a dynamic viscosity $\eta$ at a certain temperature $T$ under the influence of a force due to an electric field gradient. The analysis of motion of an ion in a viscous medium allowed Stokes to associate the values of the limiting equivalent conductivities of ions $\lambda_{0 \pm}$ with their radius and diffusion coefficient of ion $D_{0 \pm}$ in infinitely diluted solutions:

$$
\begin{gathered}
\lambda_{0 \pm}=|q| F /(6 \pi \eta \cdot r), \quad D_{0 \pm}=k T \lambda_{0 \pm} /(|q| F), \\
D_{0 \pm}=k T /(6 \pi \eta \cdot r) .
\end{gathered}
$$

Here $k$ is the Boltzmann constant. These ratios make it possible to estimate the hydrodynamic (Stokes) radius of ions using conductometric and diffusion data. Relations (7) in the form

$$
R_{\mathrm{M}}=\alpha e N \cdot F /\left(6 \pi \eta \lambda_{\mathrm{M}}\right) \quad D_{\mathrm{M}}=k T \lambda_{\mathrm{M}} /(\alpha N \cdot F)
$$

can also be applied to micelles to estimate their radius, diffusion coefficient, as well as to estimate possible values of equivalent conductivity of micelles $\lambda_{\mathrm{M}}$ or the part that relates to a charge unit $[7,8]$. In our opinion the transition to the molar conductivities of micelles is unjustified, since experimental conductivity data correlate with the total concentration of dissolved surfactant, and micelle formation causes a sharp change in the number of structural units of the dissolved substance (the total number of moles are changed).

\section{Calculation of the degree of micelles ionization and their equivalent conductivity}

In many works $[7,9-13]$ the possibilities of approximating the straight-line section of conductivity dependence by mathematical equations (5) and (6) are used to calculate the degree of micelle ionization from conductometric data. However, the available relations.

$$
a=\lambda_{+}+\lambda, \quad b=\alpha\left(\lambda_{\mathrm{M}}+\lambda_{+}\right),
$$


don't enable to carry out an analytical calculation of the degree of micelle ionization and their equivalent electrical conductivity using the coefficients $a$ and $b$ found in the experiment. An exception is the zero approximation in which the mobilities of micelles and surfactant ions are considered small compared to counterions mobilities and then $\alpha=b / a$ [11].

An additional relation for calculating the parameter $\alpha$ can be relation that equates the volume of micelle to the sum of the $N$ volumes of surfactant ions in its composition. Its combined use with equations (7) and (8) allows us to derive a formula relating the conductivity of ions and micelles

$$
\lambda_{\mathrm{M}}=\alpha \lambda_{-} N^{2 / 3} \text {. }
$$

Relations (9) and (10) lead to a quadratic equation for determining the degree of micelle ionization

$$
\alpha^{2} \lambda N^{2 / 3}+\alpha \lambda_{+}-b=0 .
$$

The solution of this equation is known as Evans formula $[14,15]$ :

$$
\alpha=\left[-\lambda_{+}+\left(\lambda_{+}^{2}+4 b \lambda N^{2 / 3}\right)^{1 / 2}\right]\left(2 \lambda N^{2 / 3}\right)^{-1} .
$$

We attempted to analyze why for the degree of micelle ionization slightly smaller values are always obtained from conductometric data using the Evans formula in comparison with other methods. When deriving a formula two main approximations are used. One of them is the use of hydrodynamic (Stokes) radii. The hydrodynamic sizes of surfactant ions, including the near layer of water molecules, are noticeably larger than their crystallographic sizes. However, when ions are combined into a micelle only the hydrocarbon parts of the surfactant ions are mainly associated. In addition, values related to specific concentrations are used while the basic formulas are valid under the conditions of infinite dilution. For example in the first approximation the equivalent conductivity of ions $\lambda_{+}+$is replaced by a larger limiting value $\lambda_{0+}$ which can be found in reference books. Accordingly, the error is also entered into the value of $\lambda=a-\lambda_{0+}$ which, on the contrary, is taken smaller than the real one.

To eliminate the error associated with the values of equivalent electrical conductivity of ions, and to calculate more correct values of $\lambda_{+}$and $\lambda$ in the case of any surfactant concentrations, we developed a method based on using the second approximation of the Debye Hückel - Onsager theory (the article [16] in the same issue).

Estimation of the need to use the developed methodology was carried out for a well-studied (also by the authors of this work [17-20]) anionic surfactant sodium dodecyl sulfate (SDS). Our values of SDS specific conductance measured at the temperature $T=25$ ${ }^{\circ} \mathrm{C}$ are similar to the data of [10], and at the temperature $T=40^{\circ} \mathrm{C}-$ the data of [9]. Because of the existence of small differences due to experimental errors and for the possibility of comparing the calculations performed, we decided to give preference to the indicated literary data. At $T=25^{\circ} \mathrm{C}$, the aggregation number of ions in micelle is taken equal to $N=64$ [10]. At $T=40^{\circ} \mathrm{C}$, the literature data on the aggregation numbers differ significantly $[8,9,21,22]$. We preferred the results of work [22] devoted to study of the temperature dependences of the parameters of SDS micelles, from which it follows that at a given temperature $N=57$. We used such experimental data (in $\left.\mathrm{S} \cdot \mathrm{m}^{2} \cdot \mathrm{mol}^{-1} \cdot 10^{-4}\right)$ : at $T=25^{\circ} \mathrm{C} \quad a=$ 66.7, $b=26.4$ [10]; at $T=40^{\circ} \mathrm{C} a=89.5, b=35.8$ [9].

The results of calculations of the degree of micelle ionization and their equivalent electrical conductivity in various approximations are given in the strings 1-3 of table 1.

The string 1 corresponds to the zero approximation, in which the mobilities of micelles and surfactant ions are considered small in comparison with counterions mobilities. Line 2 describes the standard use of the Evans formula case, in which $\lambda_{+}$is replaced by the larger limit value $\lambda_{0+}$. String 3 eliminates the disadvantages

\begin{tabular}{|c|c|c|c|c|c|c|}
\hline № & Model and approximation & $T^{\circ} \mathrm{C}$ & $\begin{array}{c}\lambda_{+} \cdot 10^{4} \\
\mathrm{~S} \cdot \mathrm{m}^{2} \cdot \mathrm{mol}^{-1}\end{array}$ & $\begin{array}{c}\lambda \cdot 10^{4} \\
\mathrm{~S} \cdot \mathrm{m}^{2} \cdot \mathrm{mol}^{-1}\end{array}$ & $\begin{array}{c}\lambda_{\mathrm{M}} \cdot 10^{4} \\
\mathrm{~S} \cdot \mathrm{m}^{2} \cdot \mathrm{mol}^{-1} \\
\end{array}$ & $\alpha$ \\
\hline \multirow[t]{2}{*}{1} & \multirow{2}{*}{$\begin{array}{l}\text { Conductometry, zero approximation } \\
\qquad \alpha=b / a, \quad \lambda_{\mathrm{M}}=\lambda\end{array}$} & $25^{\circ} \mathrm{C}$ & $\lambda_{+}=\lambda_{0+}=50.1$ & 16.6 & 16.6 & 0.396 \\
\hline & & $40^{\circ} \mathrm{C}$ & $\lambda_{+}=\lambda_{0+}=67.6$ & 21.9 & 21.9 & 0.400 \\
\hline \multirow[t]{2}{*}{2} & \multirow{2}{*}{$\begin{array}{l}\text { Conductometry, first approximation } \\
\qquad \lambda_{+} \rightarrow \lambda_{0+}, \lambda \rightarrow a-\lambda_{0_{+}}\end{array}$} & $25^{\circ} \mathrm{C}$ & $\lambda_{+}=\lambda_{0_{+}}=50.1$ & 16.6 & 62.2 & 0.235 \\
\hline & & $40^{\circ} \mathrm{C}$ & $\lambda_{+}=\lambda_{0+}=67.6$ & 21.9 & 79.1 & 0.244 \\
\hline \multirow[t]{2}{*}{3} & \multirow{2}{*}{$\begin{array}{l}\text { Conductometry, second approximation, } \\
\text { region near CMC }\end{array}$} & $25^{\circ} \mathrm{C}$ & $\lambda_{+}=46.4$ & 20.3 & 72.1 & 0.222 \\
\hline & & $40^{\circ} \mathrm{C}$ & $\lambda_{+}=62.7$ & 26.8 & 92.1 & 0.232 \\
\hline \multirow[t]{2}{*}{4} & \multirow{2}{*}{$\begin{array}{l}\text { Conductometry using micelle radius, first } \\
\text { approximation } \\
\qquad \lambda_{+} \rightarrow \lambda_{0+}, \lambda_{-} \rightarrow a-\lambda_{0+}\end{array}$} & $25^{\circ} \mathrm{C}$ & $\lambda_{+}=\lambda_{0+}=50.1$ & 16.6 & 60.9 & 0.238 \\
\hline & & $40^{\circ} \mathrm{C}$ & $\lambda_{+}=\lambda_{0+}=67.6$ & 21.9 & 76.2 & 0.249 \\
\hline \multirow[t]{2}{*}{5} & \multirow{2}{*}{$\begin{array}{l}\text { Conductometry using micelle radius, } \\
\text { second approximation, } \\
\text { region near CMC }\end{array}$} & $25^{\circ} \mathrm{C}$ & $\lambda_{+}=46.4$ & 20.3 & 62.2 & 0.243 \\
\hline & & $40^{\circ} \mathrm{C}$ & $\lambda_{+}=62.7$ & 26.8 & 78.0 & 0.255 \\
\hline
\end{tabular}

Table 1. Calculation for the equivalent conductivity of SDS ions and micelles and the degree of micelle ionization in various approximations. 
associated with the discrepancy between the values of the equivalent conductivities of ions and their conductivities at real concentrations.

It should be noted that we carried out a more rigorous calculation (string 3), which, it would seem, should have improved the value of the degree of micelle ionization and bring the data closer to the value $\alpha=0.25$, and even a little more, obtained in various experiments $[23,24]$. But it did not happen in this case. The results obtained in the first approximation, are more consistent with the experimental data than the more accurately calculated results of the second approximation. This discrepancy caused us to conclude that the use of hydrodynamic (Stokes) radii for surfactant ions is incorrect and to modify the method for calculating the conductance of ions and micelles.

\section{Determination of the parameters of micelles using the value of their radius}

To eliminate incorrect use of hydrodynamic radii of surfactant ions, we developed a modified algorithm for calculating the degree of micelle ionization which uses data on the radius of micelle. Micelle radius can be found on the basis of various methods including smallangle X-ray scattering, NMR self-diffusion, etc. The radius of SDS micelle is usually obtained equal to $R_{\mathrm{M}}=$ $2.3-2.4 \mathrm{~nm}$. According to our diffusion data for $T=40$ ${ }^{\circ} \mathrm{C}$ [17], it turned out to be $R_{\mathrm{M}}=2.34 \mathrm{~nm}$. This value we further use for calculations. In deriving a new relation for calculating the degree of micelle ionization $\alpha$, along with formulas (9), we used relation (8), containing the degree of micelle ionization and their radius. So, as the initial ratios were taken

$$
\begin{gathered}
b=\alpha\left(\lambda_{\mathrm{M}-}+\lambda_{+}\right), \\
\lambda_{\mathrm{M}}=\alpha \cdot e \cdot N \cdot F /\left(6 \pi \cdot \eta \cdot R_{\mathrm{M}}\right)=\alpha N \cdot \lambda^{*} .
\end{gathered}
$$

For convenience the coefficient $\lambda^{*}=e F /\left(6 \pi \eta R_{\mathrm{M}}\right)$ was selected which is determined by the radius of the micelle and depends on the viscosity of the medium under the experimental conditions. The combined use of these relations led to a quadratic equation, the solution of which makes it possible to determine the degree of micelle ionization as

$$
\alpha=\left[-\lambda_{+}+\left(\lambda_{+}{ }^{2}+4 b \cdot \lambda^{*} \cdot N\right)^{1 / 2}\right]\left(2 \lambda^{*} \cdot N\right)^{-1}
$$

In the first approximation of the Debye - Hückel Onsager theory, instead of the equivalent electrical conductivity of $\lambda_{+}$counterions in relation (14), the table values of the equivalent electrical conductivity of counterions $\lambda_{0+}$ can be taken. In this case, the values given in the string 4 of table 1 are obtained. Note that the obtained values, although a little more correspond to the data obtained by other experimental methods, but still quite close to the values obtained by using the Evans formula.

Using the second approximation of the Debye Hückel - Onsager theory, the obtained values can be refined. In this case, in the formula (14), the non- tabulated values of the limit equivalent electrical conductivity of counterions $\lambda_{0+}$ should be used, but the values of $\lambda_{+}$calculated for the CMC region (string 5 of table 1) in accordance with the procedure described in the same issue [16]. The second approximation somewhat improved the value of the degree of micelle ionization bringing the calculated values in closer agreement with other experimental data.

Determination of the degree of micelle ionization allows us to identify the effective charge of micelles, which for both of these temperatures, as noted in [23], is almost the same and is close to $q_{\mathrm{M}}=-15 e$. The transfer of such charge results in sufficiently large values of $\lambda_{\mathrm{M}}$, i.e. micelle contribution to the solution conductivity. The calculated values of the equivalent conductivity of micelles $\lambda_{M}$ suggest that the contribution of micelles to the total conductivity of a micellar solution is impossible to ignore because of its smallness [7,8,11]. On the contrary at high concentrations the contribution of micelles exceeds the counterion contribution, being part $\lambda_{\mathrm{M}} /\left(\lambda_{\mathrm{M}}+\lambda_{+}\right)$equal to $57.3 \%$ for $T=25{ }^{\circ} \mathrm{C}$ and $55.7 \%$ for $T=40^{\circ} \mathrm{C}$.

\section{Conclusions}

Methods for calculation of specific conductance of ions and micelles and the degree of micelle ionization using conductometric data in various approximations of the Debye - Hückel - Onsager theory was considered. The analysis of the existing calculation methods was carried out to identify their drawbacks and to suggest ways of their elimination. The calculation method of the micellar parameters on the basis of conductometric data using the micellar size was modified, and a new formula for determining the degree of micelle ionization was obtained. All calculations using the modified method were performed in the first and second approximations, and the newly obtained values of the micellar parameters are in greater agreement with the results of other studies. Based on the calculations performed, it was shown that the contribution of micelles to the total conductivity of a micellar solution cannot be neglected, since at high concentrations the contribution of micelles exceeds the contribution of counterions and can be more than $50 \%$.

\section{References}

[1] A. Toor, T. Feng, T.P. Russell, Eur. Phys. J. E Soft Matter 39(5), 57 (2016)

[2] S. Shi, T.P. Russell, Adv. Mater 30(44), e1800714 (2018)

[3] B. Saravanakumar, C. Radhakrishnan, M. Ramasamy, R. Kaliaperumal, A.J. Britten, M. Mkandawire, Results in Physics 102185, 13 (2019)

[4] P.C. Hiemenz, R. Rajagopalan, Principles of Colloid and Surface Chemistry (Boca Raton: CRC Press) (1997)

[5] V.S. Bagotsky, Fundamentals of Electrochemistry (New York: John Wiley \& Sons) (2005) 
[6] P. Atkins, J. de Paula, Physical Chemistry (New York: Oxford University Press) (2008)

[7] K.D. Danov, P.A. Kralchevsky, and K.P. Ananthapadmanabhan, Adv. Colloid Interface Sci. 206, 17 (2014)

[8] K.D. Danov, E.S. Basheva, P.A. Kralchevsky, K.P. Ananthapadmanabhan, A. Lips, Adv. Colloid Interface Sci. 168, 50 (2011)

[9] M. Benrraou, B.L. Bales, R. Zana, J. Phys. Chem. B 13432, 107 (2003)

[10] W. Al-Soufi, L. Piñeiro, M. Novo, J. Colloid Interface Sci. 370, 102 (2012)

[11] P. Carpena, J. Aguiar, P. Bernaola-Galvan, C.C. Ruiz, Langmuir 18, 6054 (2002)

[12] N. Jalšenjak, D. Težak, Chem. Eur. J. 10 5000 (2004)

[13] E. Dutkiewicz, A. Jakubowska, Colloid Polym Sci 280, 1009 (2002)

[14] L. Sepirlveda, J. Cortes, J. Phys. Chem. 5322, 89 (1985)

[15] H.C. Evans, 1956 J. Chem. Soc. 579-586

[16] O.S. Zueva, Modified method of conductometric data analysis to calculate the conductivity of surfactant ions (in the same issue)

[17] O.I. Gnezdilov, Y.F. Zuev, O.S. Zueva, K.C. Potarikina, O.G. Us'yarov, Appl. Magn. Reson. 40, 91 (2011)

[18] Yu.F. Zuev, O.I. Gnezdilov, O.S. Zueva, O.G. Us'yarov, Colloid Journal 73, 59 (2011)

[19] B.Z. Idiyatullin, K.S. Potarikina, Y.F. Zuev, O.S. Zueva, O.G. Us'yarov, Colloid Journal 75, 532 (2013)

[20] A.T. Gubaidullin, I.A. Litvinov, A.I. Samigullina, O.S. Zueva, V.S. Rukhlov, B.Z. Idiyatullin, Y.F. Zuev, Russ. Chem. Bull. 65, 158 (2016)

[21] F.H. Quina, P.M. Nassar, J.B.S. Bonilha, B.L. Bales, J. Phys. Chem. 17028, 99 (1995)

[22] B. Hammouda, Journal of Research of the National Institute of Standards and Technology 118(151) (2013)

[23] P.C. Shanks, E.I. Franses, J. Phys., Chem. 96, $1794(1992)$

[24] J.V. Joshi, V.K. Aswal, P. Bahadur, P.S. Goyal, Current Science 83(1), 47 (2002) 